\title{
RASID: A Robust WLAN Device-free Passive Motion Detection System
}

\author{
Ahmed E. Kosba \\ Dept. of Comp. and Sys. Eng. \\ Faculty of Engineering \\ Alexandria University, Egypt \\ ahmed.kosba@alexu.edu.eg
}

\author{
Ahmed Saeed \\ Dept. of Comp. Sc. and Eng. \\ Egypt-Japan Univ. of Sc. and \\ Tech. (E-JUST), Egypt \\ ahmed.saeed@ejust.edu.eg
}

\author{
Moustafa Youssef \\ Dept. of Comp. Sc. and Eng. \\ Egypt-Japan Univ. of Sc. and \\ Tech. (E-JUST), Egypt \\ moustafa.youssef@ejust.edu.eg
}

\begin{abstract}
WLAN Device-free passive ( $D f P)$ indoor localization is an emerging technology enabling the localization of entities that do not carry any devices nor participate actively in the localization process using the already installed wireless infrastructure. This technology is useful for a variety of applications such as intrusion detection, smart homes and border protection.

We present the design, implementation and evaluation of $R A S I D$, a $D f P$ system for human motion detection. $R A S I D$ combines different modules for statistical anomaly detection while adapting to changes in the environment to provide accurate, robust, and low-overhead detection of human activities using standard WiFi hardware. Evaluation of the system in two different testbeds shows that it can achieve an accurate detection capability in both environments with an F-measure of at least 0.93 . In addition, the high accuracy and low overhead performance are robust to changes in the environment as compared to the current state of the art DfP detection systems. We also relay the lessons learned during building our system and discuss future research directions.
\end{abstract}

\section{Keywords}

Anomaly detection, device-free passive localization, motion detection systems, robust device-free localization.

\section{INTRODUCTION}

The increasing need for context-aware information and the rapid advancements in communication networks have motivated significant research effort in the area of location-based services. This effort resulted in the development of many location determination systems, including the GPS system [1, ultrasonic-based systems [2, infrared-based (IR) systems [3], and radio frequencybased (RF) systems [4. Moreover, motion detection systems, that aim at detecting the motion of an entity carrying a device, were also developed [5-13. These systems require the tracked entity to carry a device that participates in the localization process. Thus, we refer to them as device-based systems.

Motivated by the wide use of wireless LANs for indoor

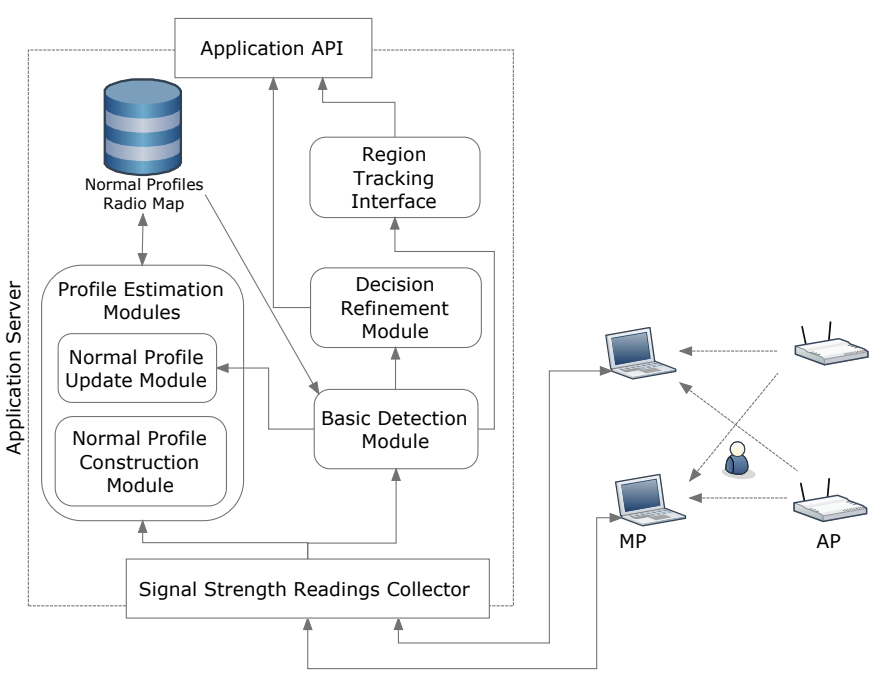

Figure 1: RASID system architecture.

communication, we recently introduced the concept of device-free passive DfP localization [14] which enables the detection and tracking of entities that do not carry any devices nor participate in the localization process. This concept depends on the fact that the presence and motion of entities in an RF environment affects the RF signal strength, especially when dealing with the 2.4 $\mathrm{GHz}$ band which is used in different IEEE standards such as $802.11 \mathrm{~b}$ and $802.11 \mathrm{~g}$ (WiFi). Different DfP algorithms were proposed for detection [14,15] and tracking 14, 16, 18 of entities in indoor environments. Our focus in this paper is on the detection problem.

In particular, we address the problem of designing a low-overhead, accurate, and robust $D f P$ motion detection system. We introduce the RASID system that provides a software only solution on top of the already installed wireless networks enabling a wide set of applications including intrusion detection, border protection, and smart homes. As a typical DfP system, RASID consists of signal transmitters, such as access points (APs), signal receivers or monitoring points (MPs), such 
as standard laptops 1 , and an application server which collects and processes information about the received signals from each MP. The application server contains the main system modules responsible for performing the detection function (Figure 1).

Our research on $R A S I D$ is motivated by several factors: First, the technologies that can be used to provide the desired detection capability (e.g. cameras [19], IR sensors, radio tomographic imaging [20, pressure sensors [21], etc) share the requirement of installing special hardware. In addition, cameras and IR sensors are limited to line-of-sight vision and thus the cost of covering an area might be prohibitive. Moreover, regular cameras fail to work in the dark or in the presence of smoke. $R A S I D$ avoids these drawbacks by using the already installed wireless infrastructure without installing any special hardware. It also makes use of the fact that RF waves do not require LOS for propagation.

From another perspective, the previously proposed WLAN DfP detection techniques 14,15 provide good performance under strong assumptions, which limit their application domain. For example, they are not robust to changes in the environment. That is they do not adapt to changes in the environment, e.g. humidity and temperature changes. Moreover, their parameters need to be changed as the deployment area changes. In addition, the technique proposed in [15] requires the construction of a human motion profile which leads to high overhead inside large-scale environments. The cost of this technique may be prohibitive, as it requires access to all areas of a building which might include restricted or private areas and requires several hours of calibration. Finally, all techniques were either evaluated in controlled environments, e.g. [14], or in small-scale real environments, e.g. 15.

In order to achieve its objectives, $R A S I D$ uses a statistical anomaly detection technique to detect motion inside indoor environments. $R A S I D$ only constructs a non-parametric profile for the signal strength readings received at the MPs when there is no human activity during a short training phase of only two minutes, leading to minimal deployment overhead. RASID also employs techniques for continuously updating its silence profile to adapt to the environment changes. The system also applies a decision refinement procedure in order to reduce the false alarms due to the signal noise. Furthermore, RASID also provides an interface by which the regions of activity can be identified. We evaluate the system in two different large-scale environments rich in multi-path and compare $R A S I D$ to the state-of-theart $D f P$ detection techniques [14,15. Our results show that $R A S I D$ achieves its goals of high accuracy in both environments with minimal deployment overhead. In

\footnotetext{
${ }^{1}$ Note that it is also possible to use the access points themselves as monitoring points.
}

addition, it is robust to changes in the environment.

In summary, the contributions of this paper are fourfold:

- We present the architecture and implementation of $R A S I D$ : a system that provides robust device-free motion detection along with techniques for adapting to environment changes and handling the wireless signal noise.

- We analyze different signal strength features that can be used for detection and identify the most promising one.

- We evaluate the system in two different large-scale real testbeds and compare it to the state-of-the-art DfP detection techniques.

- We present a comparison of parametric and nonparametric approaches for system operation.

The rest of this paper is organized as follows: Section 2 reviews related work. Section 3 presents the $R A S I D$ system architecture and operation. Section 4 presents the experimental evaluation of $R A S I D$ and a comparison with other techniques. Section 5 compares the nonparametric approach used in the system to a parametric analytical model for the system operation. In Section 6. we discuss our experience with $R A S I D$ and present some open research issues for future work. Finally, Section 7 concludes the paper and discusses future work.

\section{RELATED WORK}

Motion Detection in device-based systems has been an active field of research. Several works have been proposed to detect the motion of an entity carrying a device either with the use of special hardware like accelerometers or motion sensors [5], or by using the existing network infrastructures like wireless networks 9-11] and GSM [12,13].

From the device-free perspective, multiple technologies can be used to provide the desired capabilities including: ultra-wide band radar 22, computer vision [19, physical contact based systems [21] and radio tomographic imaging 20. Other technologies include the usage of wireless sensors for tracking transceiver-free objects 23] as well as the usage of RFID tags 24]. Those technologies share the requirement of installing special hardware to handle the device-free different functionalities. In addition, cameras and IR sensors are limited to line-of-sight vision and thus they require a high cost deployment to cover all site regions. Moreover, regular cameras can fail to work in the dark or in the presence of smoke, and they can cause privacy concerns. Ultrawide band radar based techniques also suffer from high complexity. Moreover, some techniques can require high density to provide full coverage like radio tomographic 
imaging and physical contact based systems using pressure sensors.

WLAN device-free passive systems try to avoid the above drawbacks by using the already available wireless infrastructure. The concept of device-free passive detection and tracking using WLANs was first proposed in 14 with a large number of applications including intrusion detection, border protection [25], smart homes, and traffic estimation 26]. Techniques for DfP detection [14, 15] and tracking [14,16, 17] were introduced. The proposed techniques for the detection capability are either based on time-series analysis like the moving average and moving variance techniques proposed in [14] or based on classification using the maximum likelihood estimation [15].

In comparison, RASID uses anomaly detection techniques to identify the deviations from the normal (silence) state. RASID system uses a semi-supervised statistical technique that models the learned normal behavior using a kernel-function based non-parametric estimation. The kernel-function based anomaly detection has been used in several applications where the distribution of the normal behavior is not known. For example, non-parametric estimation using Gaussian kernels was used in network intrusion detection [27] and novelty detection applied to oil flow data [28. Also, density estimation using Epanechnikov kernels was used in online outlier detection in sensor data 29] and to achieve continuous adaptive outlier detection on distributed data streams 30.

Compared to the previously proposed WLAN DfP detection techniques, the usage of the statistical anomaly detection technique, along with the other techniques devised for adapting to environment changes and refining the decision, enable $R A S I D$ to achieve low deployment overhead, high accuracy and high robustness.

\section{THE RASID SYSTEM}

In this section, we give the details of the RASID system. We start by an overview of the system architecture followed by the details of the system modules.

\subsection{System Overview}

Figure 1 gives an overview of the system architecture. The modules of the proposed system are implemented in the application server that collects samples from the monitoring points and processes them. The system works in two phases: 1) A short offline phase, during which the system studies the signal strength values when no human is present inside the area of interest to construct what we call a "normal or silence profile" for each stream. The profiles of all streams are constructed concurrently in that short phase. 2) A monitoring phase, in which the system collects readings from the monitoring points and decides whether there is hu- man activity (anomalous behavior) or not based on the information gathered in the offline phase. It also updates the stored normal profile so that it can adapt to environment changes. Finally, a decision refinement procedure is applied to further enhance the accuracy.

The Normal Profile Construction Module constructs the initial silence profiles based on a short, typically two minutes, training sample taken when there is no human motion present in the area of interest. (Section 3.3.)

The Basic Detection Module examines each stream readings in the monitoring phase and decides whether there is an anomalous behavior or not. This operation is applied to each stream independently. It also assigns an anomaly score to each stream to express the intensity of the anomalous behavior. (Section 3.5)

The Normal Profile Update Module updates the normal profiles constructed in the offline phase in order to adapt to changes in the environment. (Section 3.6)

The Decision Refinement Module applies heuristic methods to refine the decision generated by the basic detection module to reduce the false alarm rates. (Section 3.7)

The Region Tracking Interface provides an interface that visualizes the output of the above modules. This interface enables the user to identify the detected events and provides the regions of the moving entities. (Section 3.8)

We start by giving the mathematical notations followed by the details of the different modules.

\subsection{Mathematical Notations}

Let $k$ be the number of streams, which is equal to the number of APs times the number of MPs. Let $s_{j, t}$ denote the received signal strength (RSS) reading for a stream $j$ that is received at a time instant $t$. The system studies the behavior of a sliding window $W_{j, t}$ of size $l$ that ends at time $t$, i.e. $W_{j, t}=\left[s_{j, t-l+1}, s_{j, t-l+2}, \ldots, s_{j, t}\right]$.

In order to study the behavior of the sliding windows, each sliding window $W_{j, t}$ is mapped to a single feature or value $x_{j, t}$ through a function $g$. For example, if the mean is the selected feature, then $g\left(W_{j, t}\right)=$ $\frac{1}{l} \sum_{i=1}^{l} s_{j, t-l+i}$. Two types of features can be considered: measures of central tendency, such as the mean, and measures of dispersion or variation, such as the variance.

\subsection{Normal Profile Construction}

The purpose of the Normal Profile Construction Module is to construct a normal profile, capturing the received signal strength characteristics when there is no human in the area of interest. This is used later by other modules to detect anomalies. This module runs in the offline phase. It extracts the feature values from the sliding windows over the collected data and estimates its distribution. The density function of the feature val- 
ues observed is estimated using non-parametric kernel density estimation 2. This is done for each stream independently. Figure 2 illustrates the operation.

Formally, for a stream $j$, given a set of $n$ sliding windows, each of length $l$ samples, each window $W_{j, i}$ is mapped to a value $x_{j, i}$, where $x_{j, i}=g\left(W_{j, i}\right)$. Assume $f_{j}$ is the density function representing the distribution of the observed $x_{j, i}$ 's, then given a random sample $x_{j, 1}, x_{j, 2}, \ldots, x_{j, n}$, the estimated density function $\hat{f}_{j}$ is given by [31:

$$
\hat{f}_{j}(x)=\frac{1}{n h_{j}} \sum_{i=1}^{n} V\left(\frac{x-x_{j, i}}{h_{j}}\right)
$$

where $h_{j}$ is the bandwidth and $V$ is the kernel function. The choice of the kernel function is not significant for the results of the approximation 32 . Hence, we choose the Epanechnikov kernel as it is bounded and efficient to integrate:

$$
V(q)= \begin{cases}\frac{3}{4}\left(1-q^{2}\right), & \text { if }|q| \leq 1 \\ 0, & \text { otherwise }\end{cases}
$$

Also, we used Scott's rule to estimate the optimal bandwidth 32:

$$
h_{j}^{*}=2.345 \hat{\sigma}_{j} n^{-0.2}
$$

where $\hat{\sigma}_{j}$ is an estimate for the standard deviation for the $x_{j, i}$ 's.

After estimating the density function for the feature values extracted from the sliding windows, critical bounds are selected so that if the feature values observed in the monitoring state exceed those bounds, the observed values are considered anomalous. Given a significance parameter $\alpha$ and assuming $\hat{F}_{j}$ is the CDF of distribution shown in Equation [1, if the feature is a measure of central tendency, which can deviate to the left or the right, then lower and upper bounds will be calculated such that the lower bound is $\hat{F}_{j}^{-1}(\alpha / 2)$ and the upper bound is $\hat{F}_{j}^{-1}(1-\alpha / 2)$. However, if the feature is a measure of dispersion, which can only deviate in the positive (or right) direction, then an upper bound is only needed and is equal to $\hat{F}_{j}^{-1}(1-\alpha)$. In the next subsection, we study different features that can be selected.

\subsection{Feature Selection}

As the system requires an offline phase before operation, to learn the behavior of the signal readings in the normal state, the selected feature for system operation should be resistant to possible environmental changes

\footnotetext{
${ }^{2}$ In Section 5 we present the motivation for using a nonparametric approach by providing a performance comparison with a parametric modeling of the system operation.
}

that may affect the stored data, e.g. temporal variations. In addition, the selected feature should also be sensitive to the human motion to enhance the detection accuracy.

In this section, we compare two categories of features: central tendency measures and dispersion measures. The goal of this study is to identify the category that will be more promising for the system operation. For this study, we consider the mean as a central tendency measure, and the standard deviation as a measure of dispersion. We use the standard deviation, rather than the variance, as the variance is a squared measure, while the mean is not.

\subsubsection{Sensitivity to human activity}

The selected feature should be sensitive to human activity. To compare the two features, we use the Euclidean distance between the normalized histograms representing the silence and motion states. The Euclidean distance is defined as the square root of the sum of the squared distance between each corresponding histogram bin. The histograms are constructed over a two-minute period for each state using Testbed 1, which is discussed later in Section 4. Figure 3 shows the comparison versus different window sizes. The figure shows that the distance between the histograms of the standard deviation is larger than the distance between the histograms of the mean. This indicates that the standard deviation feature is more discriminant of the human motion than the mean feature. This conclusion can be justified by observing the motion effect on typical wireless signals. Figure 4 provides a visualization of the raw signal strength for two different streams during silence and human motion periods. The figure shows that in the case of human motion, the fluctuations can be up or down around the normal/silence signal level, which leads to a limited effect on the mean as compared to the standard deviation.

\subsubsection{Resistivity to temporal variations}

As the proposed system requires a learning phase before operation, it is necessary to reduce the temporal variation effect on the stored profiles. To compare the two features, we use two different silence data sets collected two weeks apart. Figure 5 shows the results. The more similar the histograms, the more resistive the feature is to the introduced variations. The figure shows that the standard deviation feature is less affected by temporal variations. This is due to the fact that the standard deviation is a relative measure as it is calculated with respect to the mean, whereas the mean itself

\footnotetext{
${ }^{3}$ Our experiments show that the changes in the traffic load on the network do not affect the signal strength. Therefore, temporal variations here refer to changes in the physical environment that affect the signal strength.
} 


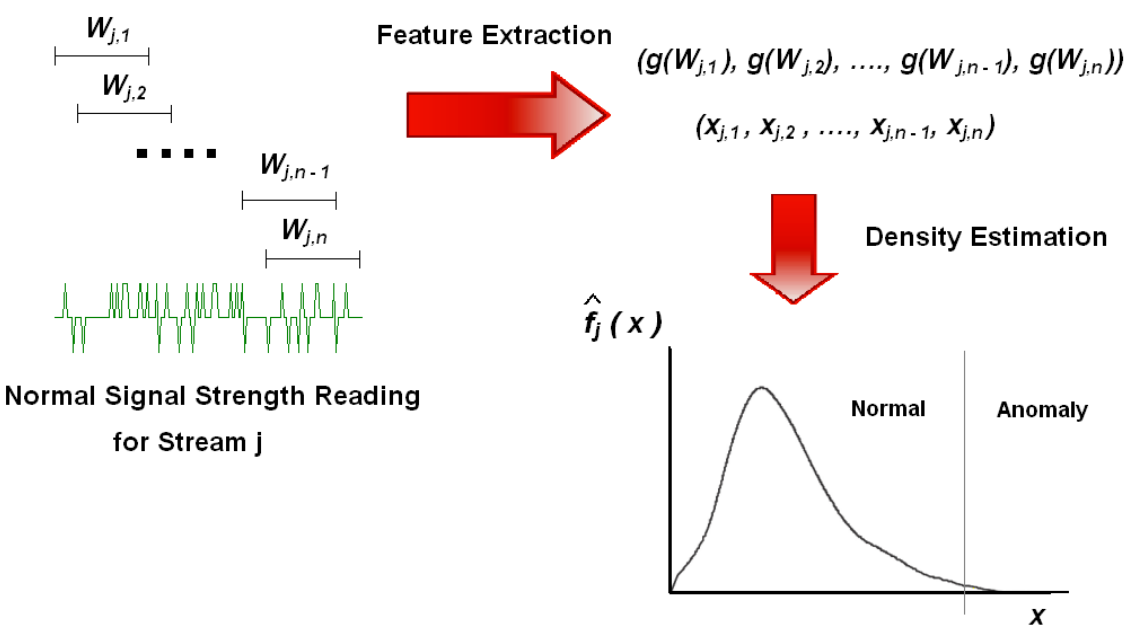

Figure 2: Illustration of the normal profile construction.

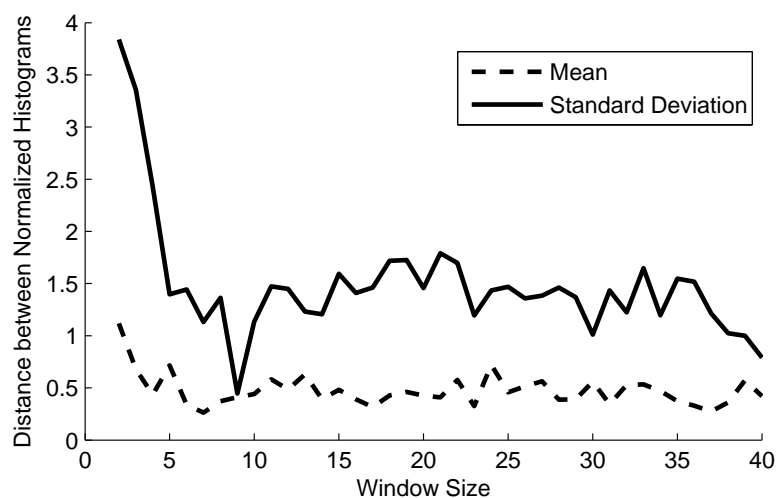

Figure 3: Distance between the features' normalized histograms for silence and motion states.

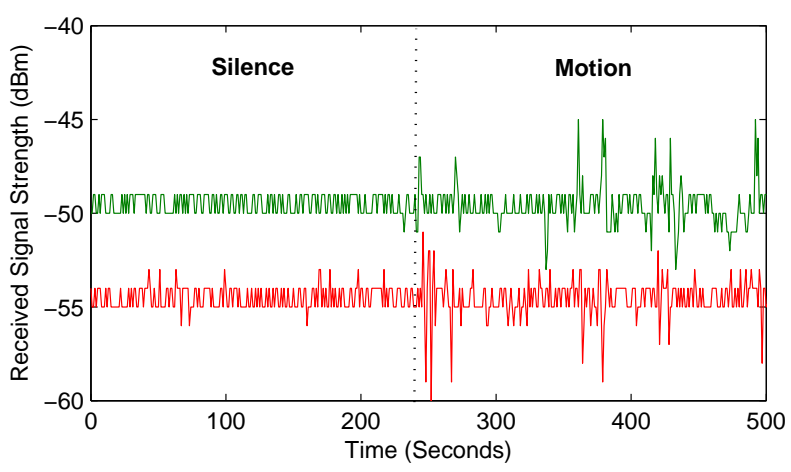

Figure 4: Illustrating the motion effect on wireless signals of two different streams.

provides an absolute value that is more susceptible to be affected by changes in the conditions.

From this study, we conclude that the measures of

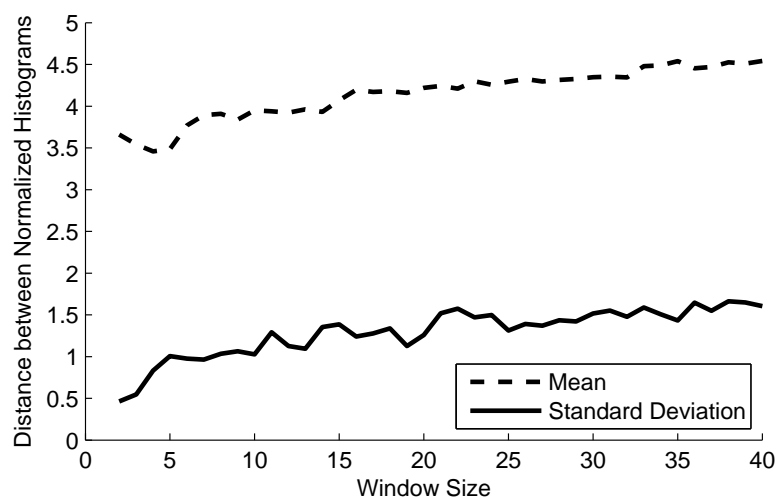

Figure 5: Distance between the features' normalized histograms for the two week-separated silence data.

dispersion, e.g. the standard deviation or variance, are more suitable for our proposed system. For the rest of the paper, we use the sample variance as the selected feature.

\subsection{Basic Detection Procedures}

The Basic Detection Module runs during the monitoring phase. The purpose of this module is to detect signal strength anomalies, i.e. human presence, based on the normal profiles constructed during the offline phase. In particular, for a window of samples $W_{j, t}$ for stream $j$ at a given time instant $t$, the module calculates the corresponding feature value $x_{j, t}$, i.e. the sample variance. A stream $j$ is considered anomalous if $x_{j, t}$ is above a critical bound $u_{j}$. Given a significance parameter $\alpha$ and assuming $\hat{F}_{j}$ is the CDF of distribution shown in Equation 1 the upper bound $u_{j}$ will be equal to the $100(1-\alpha)^{t h}$ percentile of the CDF function, such 
that $u_{j}=\hat{F}_{j}^{-1}(1-\alpha)$.

The Basic Detection Module declares a global alarm when any stream is anomalous. This approach can lead to many false positives due to signal strength outliers. This is enhanced later by the Decision Refinement Module. The Basic Detection Module also calculates an anomaly score $a_{j, t}$ for each stream $j$ to keep track of the significance of any anomalous activity. For a given window, $W_{j, t}$, the anomaly score, $a_{j, t}$, can be calculated as: $a_{j, t}=\frac{x_{j, t}}{u_{j}}$ where $x_{j, t}$ is the sample variance of the window and $u_{j}$ is the critical value. This means that a detected anomaly will have a score greater than one and a silence window will have a score of less than one. The anomaly score is used by the Normal Profile Update and Decision Refinement modules to further enhance the accuracy.

In summary, the basic detection procedure requires two parameters: the window size $l$ and the significance $\alpha$. Analysis of both parameters is presented in Section 4.3 .

\subsection{Capturing Changes in the Environment: The Normal Profile Update Module}

Due to the dynamic changes in the environment, the stored profiles may not capture the real normal state. Therefore, the systems needs to update the stored profiles during the online phase. The technique we employ for handling the update process is based on continuously updating the estimated density in Equation 10 by adding $x_{j, t}$ 's, that do not have high anomaly scores in average to it. In particular, during the monitoring phase, the system groups the consecutive $x_{j, t}$ 's in disjoint groups of size $l_{\text {update. The group that has an av- }}$ erage anomaly score of less than one is added to the normal profile. The parameter $l_{\text {update }}$ can be tuned to provide the desired performance. We quantify the effect of the $l_{\text {update }}$ parameter in detail in Section 4.3.2.

Adding new data to the normal profiles implies the need to give more weight to the recent data. Therefore, Equation 1 is modified to:

$$
\hat{f}_{j}(x)=\frac{1}{h_{j}} \sum_{i=1}^{n} w_{i} V\left(\frac{x-x_{j, i}}{h_{j}}\right)
$$

where $\sum_{i=1}^{n} w_{i}=1$. We choose linear weights such that $w_{i}=\frac{i}{n(n+1) / 2}$ ( $n$ is constant). We found that exponential weights do not provide good performance due to the high discrimination introduced between older and newer data.

\subsection{The Decision Refinement Module}

Typical wireless environments are noisy. This fact can cause many false alarms if the system generates alarms just based on a single stream. The goal of the

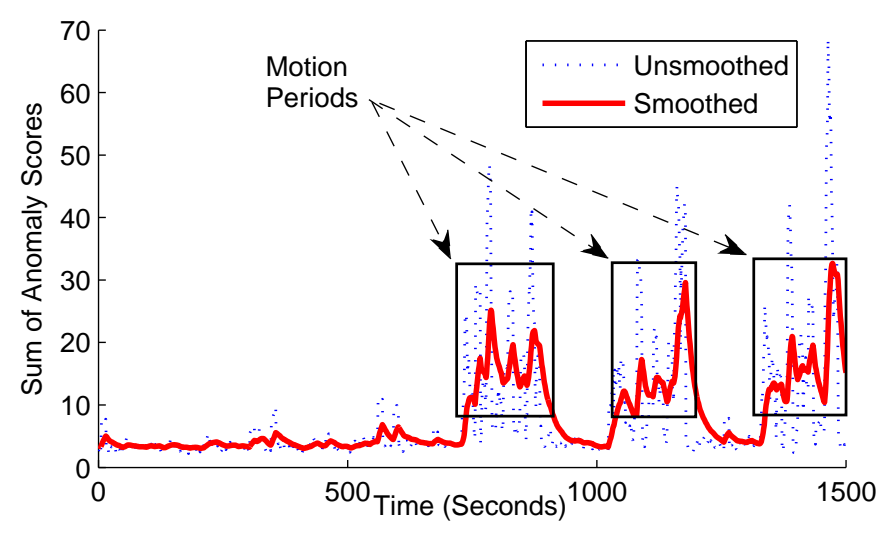

Figure 6: The behavior of the sum of anomaly scores for Testbed 1.

Decision Refinement Module is to reduce the false alarm rate by fusing different streams.

Since the Basic Detection Module assigns an anomaly score to each detected event that expresses its significance, this can be leveraged to enhance the detection performance. The Decision Refinement Module studies the behavior of a global anomaly score $a_{t}$ that is calculated by summing the individual anomaly scores for each stream. If a noticeable change in $a_{t}$ occurs, based on a threshold, while at least one stream is anomalous, this implies the start of an anomalous behavior. The module makes use of the history of the activity state inside the environment through the usage of exponential smoothing to monitor the $a_{t}$ in order to avoid the noisy samples, hence reducing the false alarm rate. It also implicitly makes use of the locality of human motion, meaning that the human will continue to affect the same stream and/or other streams near it, causing the sum of anomaly scores smoothed curve to have higher values during the motion period (Figure 6).

\subsection{Region Tracking User Interface Module}

The system provides an interface that provides information about the probable regions of the detected event. This is based on visualizing the anomaly degree of each stream enabling the user to identify the regions that probably have moving entities inside. This is done by coloring each pixel on the map according to its distance from each stream endpoints and according to the anomaly score of each stream. Figure 7 displays the output of this interface when two persons are moving inside a typical site, showing the true locations of the two persons.

\section{EXPERIMENTAL EVALUATION}

In this section, we study the effect of the different parameters on the performance of the RASID system and compare it to the previous WLAN DfP detection 


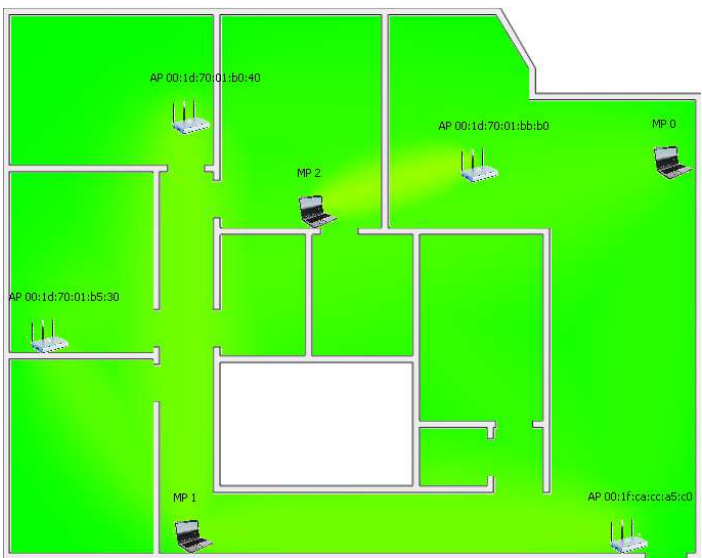

(a) Silence State

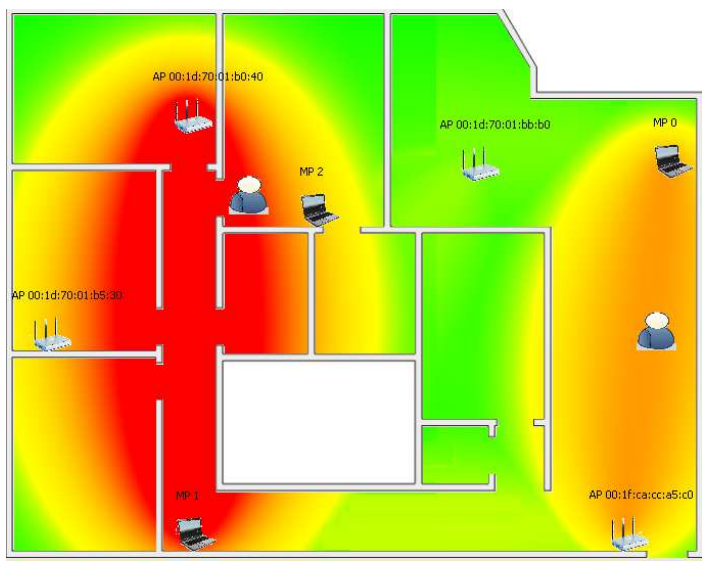

(b) Two Persons Moving

Figure 7: The output of the Region Tracking Interface.

techniques [14, 15.

\subsection{Experimental Testbeds and Data Collec- tion}

We collected two sets of data to evaluate the system performance, each in a different testbed. The first testbed is an office of approximately $2000 \mathrm{ft}^{2}$. The second experiment was conducted in a two-floor home building where each floor was approximately $1500 \mathrm{ft}^{2}$. Both tesbeds were covered with typical furniture. For both testbeds, we used four Cisco Aironet 1130AG series access points and used three DELL laptops equipped with D-Link AirPlus G+ DWL-650+ Wireless NICs as MPs. The access points were operating on different channels. The experiments were conducted in typical IEEE $802.11 \mathrm{~b}$ environments. Figures 8 and 9 show the layouts of both experiments.

For the data collection, sets of normal (silence) state readings and continuous motion readings were collected for each testbed. A total of about one hour and 15 minutes of data was collected for each testbed with a

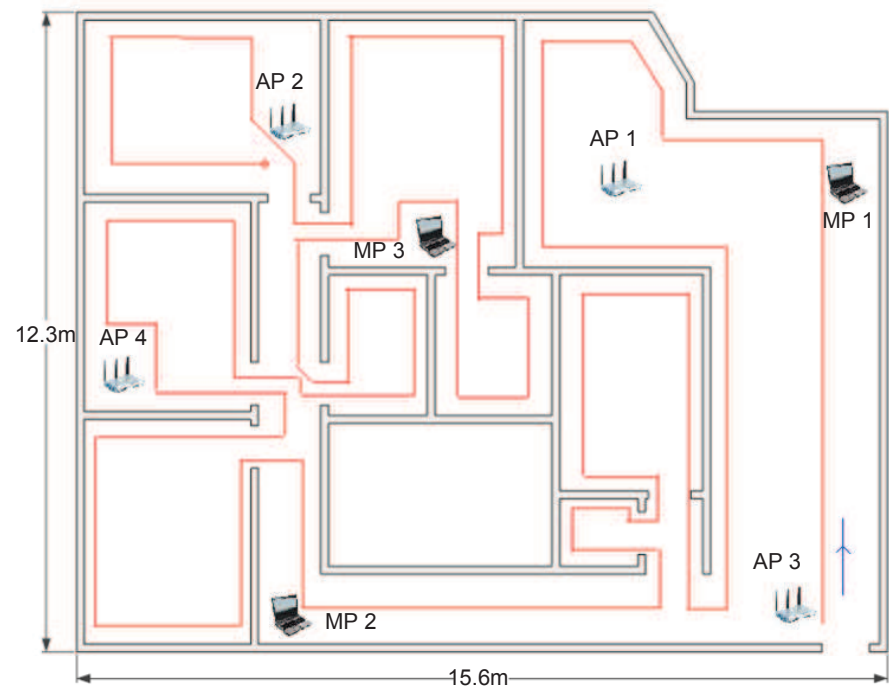

Figure 8: Testbed 1 layout and motion pattern.

sampling rate of one sample per second using the active scanning technique [4. For Testbed 1, this includes three motion sets, while for Testbed 2, this includes two motion sets. A motion set covers the entire area of the testbed, as shown in figures 8 and 9 and represents the motion of a single person walking normally around the site without any stops.

For system evaluation, extreme conditions were employed: The training period is chosen to be only the first two minutes of the entire data collected with the absence of human motion. In addition, only one person moved in the area of interest. More people in the area of interest will lead to higher variance 33 and hence better detection. Therefore, the reported results present a lower bound on the performance of the RASID system.

\subsection{Evaluation Metrics}

We used three metrics to analyze the detection performance: the false positive (FP) rate, the false negative $(\mathrm{FN})$ rate and the $\mathrm{F}$-measure. The false positive rate refers to the probability that the system generates an alarm while there is no human motion in the area of interest. The false negative rate refers to the probability that the system fails to detect the human motion in any place in the area. We also use the F-measure, which provides a single value to measure the effectiveness of the detection system [34.

Since each anomalous sample may not be detected simultaneously, we also studied the detection latency, i.e. how much time the system needs to associate an anomalous sample with a detected event. The overall $90^{t h}$ detection latency percentile in both testbeds was found to be less than one second.

\subsection{System Performance}




\begin{tabular}{|r|r|c|c|c|}
\hline & & $\begin{array}{c}\text { Basic Detection } \\
\text { Module }\end{array}$ & $\begin{array}{c}\text { Normal Profile } \\
\text { Update Module }\end{array}$ & $\begin{array}{c}\text { Decision Refinement } \\
\text { Module (RASID Perf.) }\end{array}$ \\
\hline \multirow{3}{*}{ Testbed 1 } & FN Rate & 0.0672 & 0.0876 & $\mathbf{0 . 0 4 6 8}$ \\
& FP Rate & 0.2158 & 0.1176 & $\mathbf{0 . 0 3 7 8}$ \\
& F-measure & 0.8683 & 0.8989 & $\mathbf{0 . 9 5 7 4}$ \\
\hline \hline & Enhancement & - & $3.52 \%$ & $\mathbf{1 0 . 2 6} \%$ \\
\hline \multirow{3}{*}{ Testbed 2 } & FN Rate & 0.2368 & 0.2069 & $\mathbf{0 . 0 9 6 6}$ \\
& F-measure & 0.1059 & 0.0903 & $\mathbf{0 . 0 3 7 2}$ \\
& Enhancement & - & 0.8422 & $\mathbf{0 . 9 3 1 1}$ \\
\hline
\end{tabular}

Table 1: System performance under the same parameters $\left(l=5, \alpha=0.01, l_{\text {update }}=15\right)$ for the two testbeds. Enhancement is with respect to the F-measure of the Basic Detection Module.

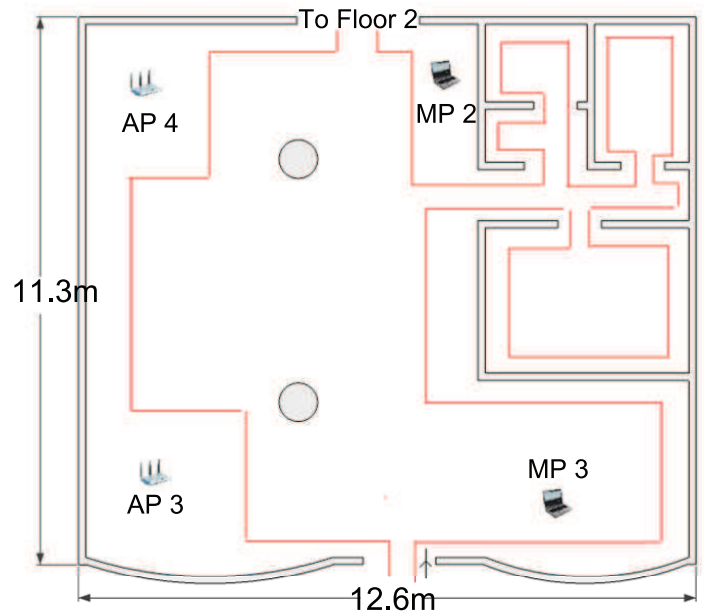

(a) Floor 1

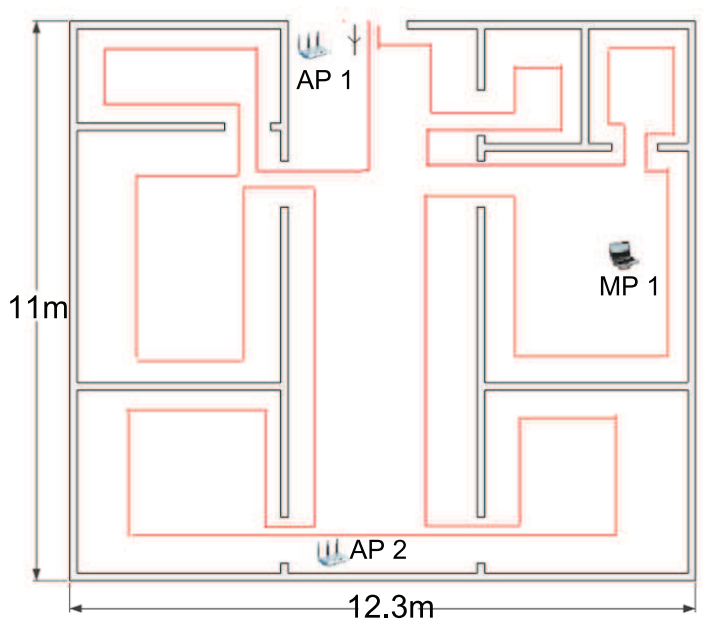

(b) Floor 2

Figure 9: Testbed 2 layout and motion patterns.

Table 1) 1summarizes the system performance for both testbeds using the same parameters for all modules. The table also shows the enhancement introduced by each module to show the robustness of the techniques.

\subsubsection{Basic Detection Module}

As mentioned earlier, this module requires the selection of the sliding window size $l$ and the significance $\alpha$. Figure 10 illustrates the effect of these parameters applied to Testbed 1. Similar performance has been observed for Testbed 2. The figure shows that choosing a too short window size will make the system less sensitive to human motion. On the other hand, choosing a very large window size will introduce a very high FP rate. For the significance parameter, as $\alpha$ decreases, the FP rate decreases and the FN rate slightly increases. This means that increasing the significance will result in less system sensitivity. Therefore, to balance the different performance metrics, we choose $l=5$ and $\alpha=0.01$.

Table 1 shows that Testbed 2 has a higher FN rate than Testbed 1 in the Basic Detection Module. This is due to the larger testbed area (i.e. less coverage) and the time needed to move between the floors in Testbed 2 . This is significantly enhanced by the processing performed by the Normal Profile Update and Decision Refinement modules. It can be noted also that the FP rate in Testbed 1 is relatively high. This is because the two-minute training period is not enough to sustain accurate detection for one hour of accurate operation inside the office environment. This highlights the need for the Normal Profile Update Module.

\subsubsection{Normal Profile Update Module}

The Normal Profile Update Module requires the selection of the update window size $l_{\text {update }}$. Choosing a too small $l_{\text {update }}$ will make the system very sensitive to noisy readings causing a high FP rate. On the other hand, a very large $l_{\text {update }}$ will make the system less sensitive to human motion causing a higher FN rate. Figure 11illustrates these effects of the update window size on the system performance for Testbed 1 when $l=5$ and $\alpha=0.01$. The figure shows that an update window size between 10 and 20 is sufficient to reduce the high FP rate without causing much increase to the FN Rate. Thus, we choose $l_{\text {update }}=15$. The results are shown in Table 1. The table shows that there is about $50 \%$ reduction in the FP rate in the first testbed, but this lead to a slight growth in the FN rate. For Testbed 2, the results of both the FN and the FP rates were en- 


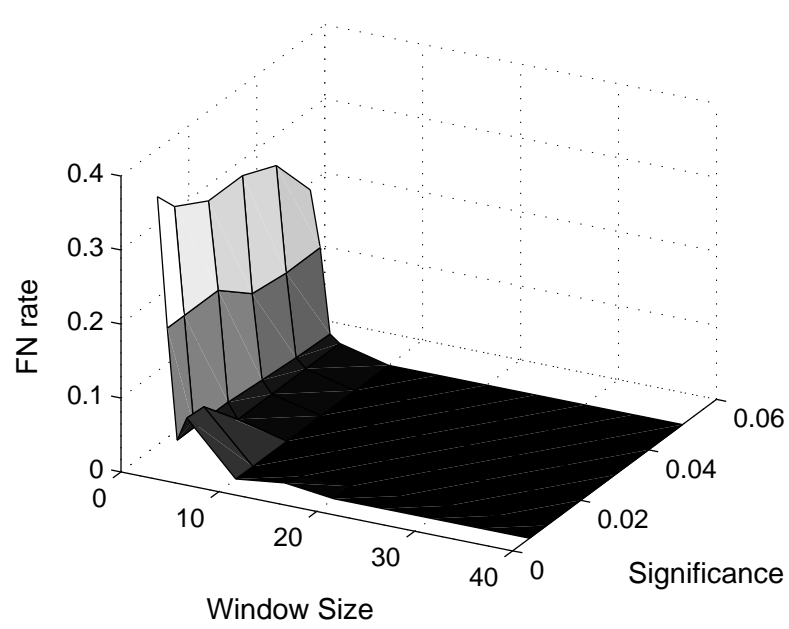

(a) False negative rate

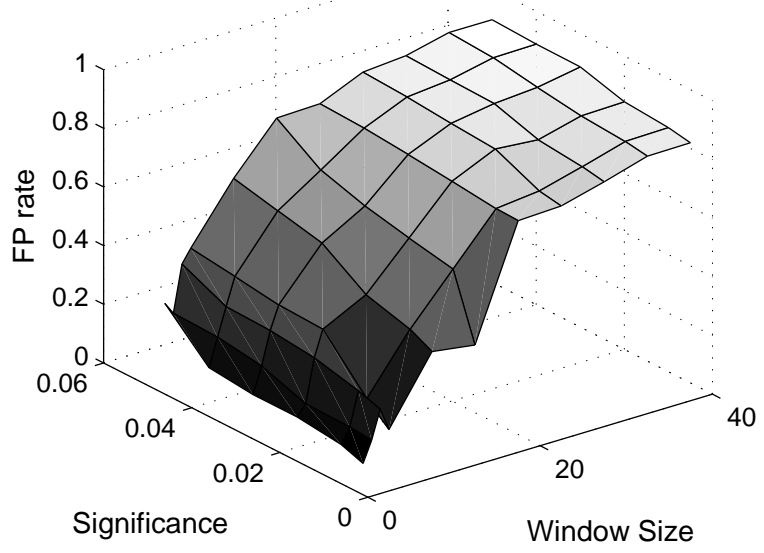

(b) False positive rate

Figure 10: Analysis of the Basic Detection Module parameters for Testbed 1.

hanced due to adapting to the environment. Overall, the F-measure was enhanced by 3 to $4 \%$ with respect to the Basic Detection Module performance.

This enhancement can be explained by the observation that the Normal Profile Update Module reduces the effect of the temporal variations between the environment true normal profiles and the stored normal profiles by updating them. We verified that by applying the two-sample Kolmogorov-Smirnov test to the distributions of the updated profiles and the distributions of the true normal state. The test accepted the hypotheses that those distributions came from the same underlying distribution at a significance of 0.05 . Figure 12 provides an example comparing the starting, updated and true sample variance profiles at the end of Experiment 1.

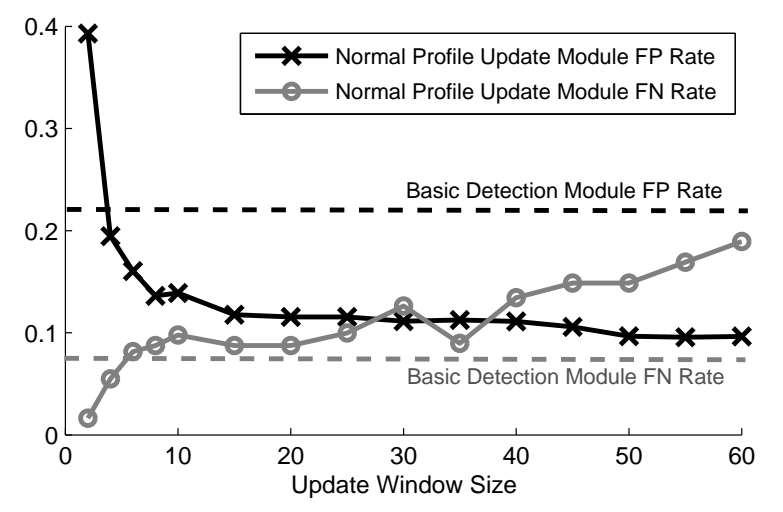

Figure 11: Effect of the update window size parameter $\left(l_{\text {update }}\right)$.

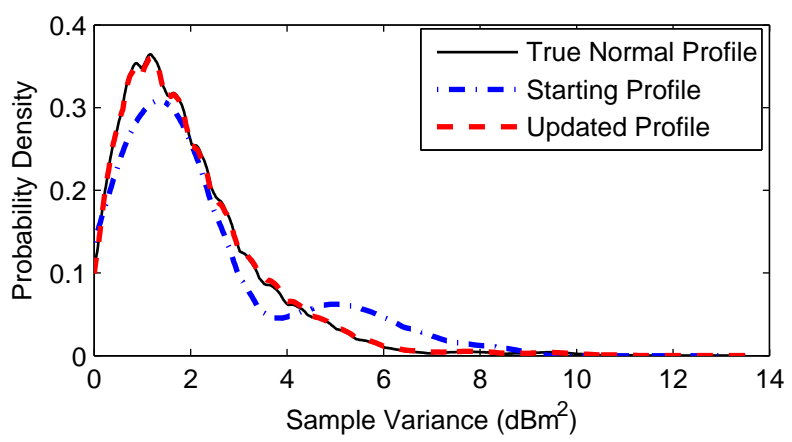

Figure 12: Comparison between the starting profile, updated profile and the true profile for the sample variance of the AP4-MP3 stream at the end of Experiment 1. As shown, the updated and true profiles are almost congruent.

\subsubsection{Decision Refinement Module}

This module fuses the data from all streams. Figure 6 displays the sum of anomaly scores curve for the data collected for Testbed 1. To reduce the FP rate, the curve is exponentially smoothed with a smoothing coefficient of 0.04. A large increment in the smoothed curve, by more than $20 \%$ to $25 \%$ from the normal level, implies a period of human motion. Our experiments show that deviations from these parameters values will not lead to significant degradation in the results. The figure shows that the motion periods are clearly distinguishable from the silence state. Table 1 shows that this module can lead to up to 10 to $14 \%$ enhancement in the F-measure for both testbeds with respect to the Basic Detection Module. It is important to note that this module also reduced the $\mathrm{FN}$ rate, as some of the previously undetected events are now detected because this technique makes use of the history of the state of the activity as described earlier.

\subsection{Comparison with Previous Techniques}


In this section, we compare the performance of $R A S I D$ to the previous techniques devised for WLAN DfP detection. We start by a brief description of the techniques, followed by the different aspects we evaluate the techniques on. Finally, we present the results of the comparison.

\subsubsection{Comparison Techniques}

Three techniques are considered for the comparison:

1. The moving average technique [14: The moving average technique uses a central tendency feature, i.e. the average. It uses two sliding window averages: a short window average representing the current system condition and a long window average representing history. The idea is to compare the two averages and if the difference is above a threshold, a detection is announced. It is important to note that the moving average technique does not require a training phase.

2. The moving variance technique 14: The moving variance technique uses a dispersion feature, i.e. the variance. Similar to the moving average technique, it compares the variance of the current system state, based on a sliding window, to the variance of the silence period, obtained through a training phase. If the difference is above a threshold, a detection is announced.

3. The maximum likelihood classification (MLE) technique [15]: This technique constructs profiles for the silence period as well as for the motions period for different locations in the area of interest. The profiles represent the signal strength distribution for each stream at each location. Therefore, it involves significant training data. During the detection phase, the system finds the profile that has the maximum likelihood given a signal strength vector, one entry for each stream. If the estimated profile corresponds to a motion profile, an alarm is generated.

\subsubsection{Comparison Aspects}

- Static accuracy: accuracy when the system is evaluated with the same profiles it was trained on (if any). This is to test the best attainable accuracy.

- Profiles' robustness: that is how consistent the performance of the system is when the tested profiles are different from the trained ones, for example due to temporal changes in the environment. For this case, the testing data set is collected two weeks after the data sets used for training.

- Overhead: the effort needed to deploy the system.

\subsubsection{Comparison Results}

Table 2 shows the comparison results in two cases.

In terms of the static accuracy, the results show that the F-measure of the RASID system is better than other systems in Testbed 1 and is slightly lower than the MLE technique in Testbed 2. Compared to the Moving Average and Moving Variance techniques, the RASID system provides high accuracy due to the techniques it uses to enhance the performance. On the other hand, the MLE technique achieves slightly higher accuracy in Testbed 2 as it stores a motion profile, which requires much higher overhead than the RASID system.

In terms of profiles' robustness, the Moving Average technique does not store any profiles. Therefore, its overall performance is low but almost the same as the profiles change. On the other hand, the robustness of the MLE technique is the least as it uses the mean signal strength values as the features used for classification. Therefore, after two weeks, the distribution of the signal strength does not follow the learned one. This is why the FP rate for the MLE technique is too high due to the shift that occurred in the signal distributions. It can also be noted that RASID performance in the two cases was the best because RASID uses the variance for its operation (dispersion feature) and employs techniques for adapting to changes in the environment and for enhancing the performance. This is why RASID performance is better than the Moving Variance in general, although the Moving Variance uses the same feature as RASID.

In terms of overhead, the Moving Average technique has the minimum overhead as it does not need any learning phase. The Moving Variance and RASID deployment need to construct normal profiles by collecting samples for two minutes when the human is not present. On the other hand, the MLE technique has the worst overhead as it constructs motion profile at each location in the area of interest in addition to the normal profile.

In summary, although the static detection accuracy of RASID is as accurate as the MLE technique, the MLE technique has significantly higher overhead than $R A S I D$ because of its motion profile requirements. In addition, RASID is the most robust technique to temporal changes in the training profiles and significantly outperforms the remaining techniques.

\section{COMPARISON WITH A PARAMETRIC APPROACH}

In this section, we compare the performance of the system's non-parametric approach to an analytical model that models the sample variance parametrically. The results of this model can help validate the results of our parameter analysis in the previous section and can also motivate the usage of the non-parametric density estimation. The next evaluation will be based on the results 


\begin{tabular}{|c|c|c|c|c|c|}
\hline & \multicolumn{5}{|c|}{ Results with static profiles } \\
\hline & & Moving Average & Moving Variance & MLE & $R A S I D$ \\
\hline \multirow{3}{*}{ Testbed 1} & FN Rate & 0.1446 & 0.1426 & 0.0363 & 0.0468 \\
\hline & FP Rate & 0.1385 & 0.104 & 0.1547 & 0.0378 \\
\hline & F-measure & 0.858 & 0.8743 & 0.9099 & 0.9574 \\
\hline \multirow{6}{*}{ Testbed 2} & FN Rate & 0.0759 & 0.308 & 0.0372 & 0.0966 \\
\hline & FP Rate & 0.7412 & 0.1478 & 0.0774 & 0.0372 \\
\hline & F-measure & 0.6935 & 0.7522 & 0.9438 & 0.9311 \\
\hline & Overhead & No overhead & Minimal & Worst & Minimal \\
\hline & \multicolumn{5}{|c|}{$\begin{array}{l}\text { Results with testing profiles separated two weeks } \\
\text { from the training profiles. }\end{array}$} \\
\hline & & Moving Average & Moving Variance & MLE & $R A S I D$ \\
\hline \multirow{3}{*}{ Testbed 1} & FN Rate & 0.2165 & 0.319 & 0.1653 & 0.0472 \\
\hline & FP Rate & 0.0711 & 0.1561 & 0.952 & 0.0782 \\
\hline & F-measure & 0.8449 & 0.7414 & 0.5991 & 0.9383 \\
\hline \multirow{4}{*}{ Testbed 2} & FN Rate & 0.2641 & 0.4152 & 0.1203 & 0.0931 \\
\hline & FP Rate & 0.3602 & 0.0513 & 0.831 & 0.0722 \\
\hline & F-measure & 0.7022 & 0.7149 & 0.6491 & 0.9165 \\
\hline & Overhead & No overhead & Minimal & Worst & Minimal \\
\hline
\end{tabular}

Table 2: Performance comparison with previous $D f P$ detection techniques.

of the Basic Detection Module only, so as to evaluate the two approaches without the enhancements. First, we describe the analytical model, then we present the results of the comparison.

\subsection{The parametric model}

The sample variance can be modeled parametrically given some conditions. According to Cochran's Theorem 35, the sample variance of $l$ independent normally distributed random samples follows a chi-square distribution with $l-1$ degrees of freedom such that $\frac{(l-1) s^{2}}{\sigma^{2}} \sim \chi_{l-1}^{2}$, where $\sigma^{2}$ is the population variance. According to $\underline{36}$, the signal strength readings (in $\mathrm{dBm}$ ) distributions for a stream $j$ can be assumed to follow a normal distribution. Given that assumption, a parametric model can be devised for the system when the sample variance is used. Given a significance $\alpha$ and a window size $l$, the upper bound for the sample variance observed during the monitoring phase is $\chi_{l-1,1-\alpha}^{2}$. However, 36 also stated that the normality assumption may not hold in some cases. In addition, the signal strength readings may not be independent [37. Thus, we believe that the non-parametric model described before will provide better performance than the parametric model. This will be verified in the following subsection.

\subsection{Analysis Results}

First, to check how close the parametric model is to the actual system, we compare the critical upper bounds obtained by both methods. For example, in Figure 13 we compare the critical sample variance values in both cases for the stream AP4-MP3 from Experiment 1 , when the population variance is assumed to be $2.02 \mathrm{dBm}^{2}$ which is an experimental estimate for the population variance of that stream. The figure shows that the parametric model and the actual system crit-

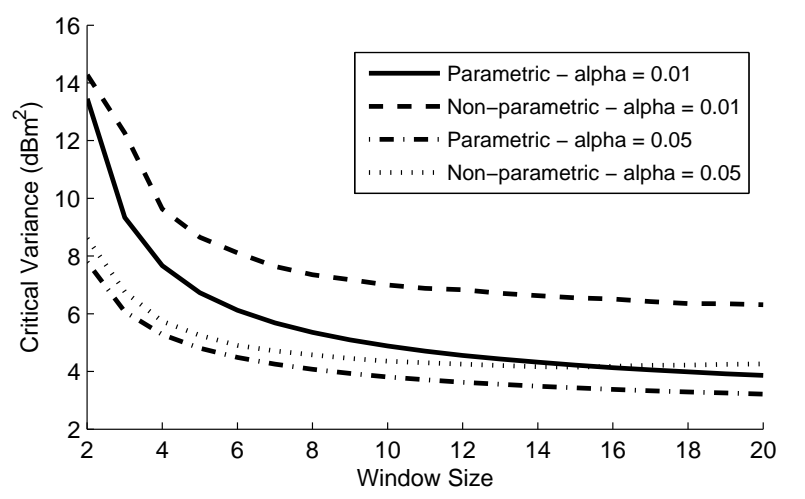

Figure 13: Comparison of the critical variance values of the parametric model and the RASID system model (non-parametric approach).

ical values follow the same trends. However the difference between the curves suggests that the real case does not exactly follow the assumed parametric model. In addition, the effects of Basic Detection Module parameters can be inferred from the parametric model curves. As the window size parameter $l$ increases, the critical variance value decreases which results in increased system sensitivity (i.e. higher FP rate and lower FN rate). Also, as the significance parameter $\alpha$ increases, the critical variance value decreases which also results in increased sensitivity. This is consistent with the analysis presented in Figure [10.

The next point is to study how the usage of the parametric model instead of non-parametric estimation can affect the system performance. As the distribution of the sample variance depends on the population variance, we analyze its effect. Figure 14 shows the effect of the population variance on the performance of the Basic Detection Module when the parametric model is 


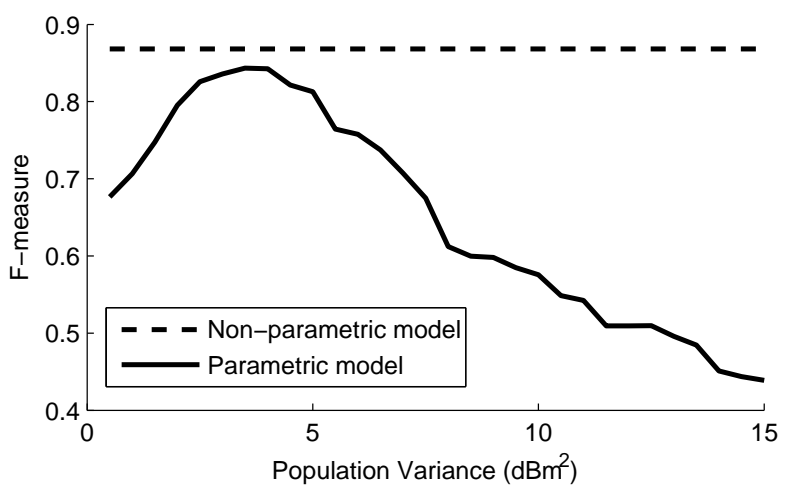

Figure 14: The performance of the Basic Detection Module when the parametric model is used versus the population variance, given $l=5$ and $\alpha=0.01$. The best population variance configuration provides an F-measure of $0.843 \mathrm{com}$ pared to 0.8683 that was obtained using the nonparametric estimation.

used for Experiment 1. From the figure, we can conclude that the best performance achieved in terms of the F-measure (0.843) is less than the F-measure obtained using non-parametric estimation (0.8683).

To conclude, the parametric model leads to lower performance compared to the non-parametric estimation because the assumptions that the signal strength values are independent and follow a normal distribution may not hold. Also, the parametric model requires the selection of an accurate population variance. This cannot be done accurately without training for long time periods. Therefore, we conclude that RASID approach of constructing non-parametric profiles in a short offline phase and updating them in the online phase does provide a better option.

\section{DISCUSSION}

In this section, we discuss some points related to the configuration and the performance of the RASID system. We also highlight some research issues and some challenges that can be addressed in future work.

\subsection{Univariate VS Multivariate Density Esti- mation}

As mentioned before, the basic detection module studies each stream independently by estimating the univariate density for the selected feature of the sliding windows extracted from the training data. Another possibility was to construct a multivariate density estimate for the data of all streams. This implies a modification to the anomaly detection criteria. Different algorithms can be applied in this case, e.g. 27. Our experience with this algorithm shows that this leads to a degradation of the system accuracy. The main rea- son for this degradation is that the system sensitivity is significantly reduced, especially when the number of streams is large. In that case, the system may not be able to detect an anomaly in one stream only, as its effect may not be much sensed.

\subsection{Effect of Network Activity on System Pro- files}

Typically in real wireless environments, it is expected that many monitoring points may be using the wireless network for handling typical tasks (e.g. downloading updates or patches). The question is whether such network activities will require any change in the system normal profiles if they were originally collected while there is no network activity. In this subsection, we present an experimental study to investigate that effect.

In order to examine that effect, a simple experiment was conducted on a single stream between an access point and a laptop acting as a monitoring point in silence state. Two signal strength data sets were collected while there was no network activity at the monitoring point, while another two sets were collected while the monitoring point were downloading data through the wireless stream with the maximum download speed allowed (50 KBytes per second). The collected data are used to construct normal profiles in the same way presented earlier in Section [3.3. Figure [15] compares the constructed profiles for the four sets. From the figure, it is clear that the difference between the distributions in both cases is negligible. Furthermore, we apply the twosample Kolmogorov-Smirnov test to each of the four different pairs of those constructed profiles. The test accepted the hypotheses that those estimated distributions came from the same underlying distribution with a significance of 0.05 . Therefore, we can conclude that the constructed sample variance profiles are invariant with respect to the state of network activity.

\subsection{Detection and Identification of Indepen- dent Events}

The above experiments showed that the system is capable of detecting a single person moving inside the area of interest. Obviously, the detection performance will be enhanced if there were more than one entity in the area of interest. We verified that the system will be able to declare that there is anomalous behavior inside the area more clearly in this case.

It would be useful to identify the number of moving entities in some applications. Figure 7 shows that we can detect that there are multiple entities in the area of interest. However, as our system uses limited data to satisfy the feasibility design goal (normal profiles only), the system cannot provide full information about the number of entities in all cases. For example, if two 


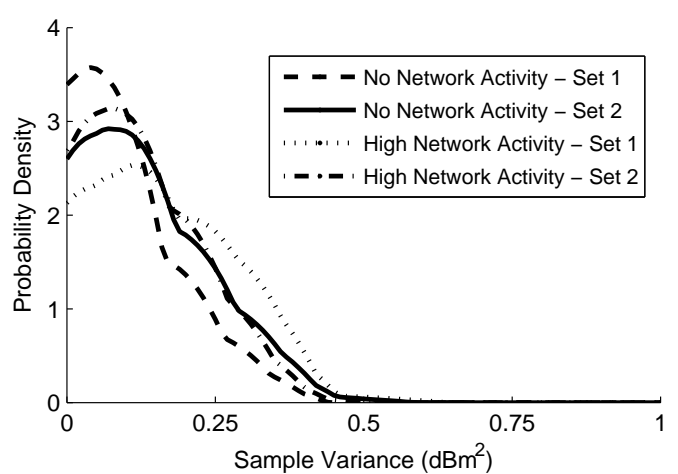

Figure 15: The effect of network activity on the constructed sample variance normal profiles. As shown, the difference between the profiles is not significant.

entities are affecting a single stream only, the system will detect them as one entity. This is because there is no enough information that enables the system to differentiate between the two cases. On the other hand, in some cases, the system can tell with high probability that some events are due to independent entities. Here, we briefly describe the constraints through which the system can provide information about the number of independent entities.

First, let $T_{\min }$, a $k \times k$ square matrix denote what we call a minimum time reachability matrix. Each entry in this matrix stores the minimum time needed for an entity to affect two streams $i$ and $j$, such that

$$
T_{m i n_{i j}}=\frac{D_{\min _{i j}}}{v_{\max }}
$$

, where $D_{\min _{i j}}$ represents the minimum distance between the nearest two points on the $i$ and $j$ streams lines of sight and $v_{\max }$ represents the maximum movement velocity inside the area. The distance $D_{\text {min }_{i j}}$ can be calculated from the site map, and $v_{\max }$ can be estimated based on empirical observations.

Two events $E_{1}$ and $E_{2}$ are considered independent (i.e. not generated by the same entity), if they satisfy the following conditions. First, they should be affecting two different streams $i$ and $j$ and second, the time difference between $E_{1}$ and $E_{2}$ is less than the value $T_{m_{i n} i j}$. The time difference between the two events are calculated based on the time difference between the times when the anomaly scores for the two events reach the peaks as they express the moments when the entities are affecting the streams the most. To tell that $n$ events are independent, each pair of those events should satisfy the conditions described above. The above conditions imply that the system cannot detect more than $k$ moving entities, where $k$ is the number of streams as stated earlier.

To conclude, despite the limited information the system uses, the system can provide information about the number of independent events inside the monitored area given some conditions. The significance of this point can be clear when applied inside large scale environments.

Another possibility is to use the level of the change in variance as an indication of the number of entities. The hypothesis is that the more human affecting a single stream, the higher the variance should be. This hypothesis still needs to be verified though.

\subsection{Integration with DfP Tracking Systems}

Our system can provide useful information to DfP tracking systems like the ones proposed in [16.17]. First, a DfP tracking system can use our system to decide whether to start the tracking process or not. Also, the system can enhance the tracking accuracy by limiting the probable locations to a certain area (e.g. as in Figure 7). In addition, given the conditions described earlier, our system can help the tracking system identify the number of intruders and the area of each one, so that it can apply the tracking algorithms to each area independently. This will need further investigation and experimentation.

\subsection{Combining Features}

Although we showed in this paper that using the variance as a feature is better than using the mean, both features can be used concurrently to achieve better performance. Our initial results show that combining both features and using a simple voting scheme can enhance the results in some cases. This is a subject for future investigations.

\subsection{Signal Strength Readings Synchronization}

The synchronization of the signal strength readings received at the monitoring point can be necessary in some cases. For example, the technique described before for checking the independence of the detected events requires synchronization of the readings across the streams. In addition, the decision refinement module requires the different streams to be synchronized. In this paper, we took a centralized approach for synchronization, where the application server requests the MPs to initiate a reading. Other approaches, such as time synchronization of the MPs can be employed. The advantages and disadvantages of each technique in terms of accuracy and overhead can also be investigated.

\subsection{Effect of Different Hardware}

The hardware used to capture the signal strength values can affect system performance. Through our experiments, we studied how the WLAN NIC type affects the quality of the collected readings. We found that NICs 
differ in two main aspects: sensitivity to human activity and noise readings. For example, some cards cannot sense the human shadowing effect unless it is sustained for a sufficient period of time. The readings of some other cards are noisy and requires extensive filtering. These experiments considered the NICs only. However this can hold from the APs perspective too. Therefore, we believe a study is needed to identify which hardware will be more suitable for the system operation and how to account for these variation between cards and allow the system to operate with different cards.

\section{CONCLUSIONS AND FUTURE WORK}

In this paper, we presented the $R A S I D$ system, a system that enables device-free passive motion detection using the already installed wireless networks. $R A S I D$ uses non-parametric statistical anomaly detection techniques to provide the detection capability. The $R A S I D$ system also employs profile update techniques to capture changes in the environment and to enhance the detection accuracy. The system was evaluated in two different real environments. Using the same parameters for the two testbeds, the system provided an accurate detection capability reaching an F-measure of at least 0.93 in both testbeds. The performance of the $R A S I D$ system was compared to the previously introduced techniques for WLAN DfP detection. The results showed that the $R A S I D$ system outperformed the state-of-theart techniques in terms of robustness and accuracy. In addition, we showed that the non-parametric approach employed by RASID has significant advantages over a parametric approach for the system operation.

Currently, we are expanding RASID in several directions: One direction is to integrate $R A S I D$ 's detection capability with $D f P$ tracking systems while considering larger testbeds. Another direction is to study possible sources of noise in typical wireless environments, e.g. other devices inside or outside the area of interest, and how to reduce their effect. We are also studying how the detected entity's characteristics, e.g. size, shape and motion pattern, can affect the system performance. Moreover, the site configuration, i.e. the positions of the APs and MPs, can also be studied in order to optimize the system performance.

\section{REFERENCES}

[1] P. Enge and P. Misra, "Special Issue on Global Positioning System," in Proceedings of the IEEE, January 1999, pp. 3-172.

[2] N. B. Priyantha, A. Chakraborty, and H. Balakrishnan, "The Cricket Location-Support System," in MobiCom '00: Proceedings of the 6th Annual International Conference on Mobile Computing and Networking, New York, NY, USA, 2000, pp. 32-43.
[3] R. Want, A. Hopper, V. Falcao, and J. Gibbons, "The Active Badge Location System." ACM Trans. Inf. Syst., vol. 10, no. 1, 1992.

[4] M. A. Youssef and A. Agrawala, "The Horus WLAN Location Determination System," in Communication Networks and Distributed Systems Modeling and Simulation Conference, 2005, pp. 205-218.

[5] J. Krumm, L. Williams, and G. Smith, "SmartMoveX on a Graph - An Inexpensive Active Badge Tracker," in UbiComp '02: Proceedings of the 4th International Conference on Ubiquitous Computing, 2002.

[6] C. Randell and H. Muller, "Context Awareness by Analyzing Accelerometer Data," in ISWC '00: Proceedings of the 4th IEEE International Symposium on Wearable Computers, 2000, pp. $175-176$.

[7] L. Bao and S. S. Intille, "Activity Recognition from User-annotated Acceleration Data," in Pervasive Computing (LNCS), vol. 3001, 2004.

[8] M. Philipose, K. P. Fishkin, M. Perkowitz, D. J. Patterson, D. Fox, H. Kautz, and D. Hahnel, "Inferring Activities from Interactions with Objects," IEEE Pervasive Computing, vol. 3, October 2004.

[9] M. Wallbaum and S. Diepolder, "A Motion Detection Scheme For Wireless LAN Stations," in Proceedings of the 3rd International Conference on Mobile Computing and Ubiquitous Networking, 2006.

[10] J. Krumm and E. Horvitz, "LOCADIO: Inferring Motion and Location from Wi-Fi Signal Strengths," 2004, pp. 4-13.

[11] K. Kleisouris, B. Firner, R. Howard, Y. Zhang, and R. P. Martin, "Detecting Intra-room Mobility with Signal Strength Descriptors," in MobiHoc '10: Proceedings of the Eleventh ACM International Symposium on Mobile Ad Hoc Networking and Computing, 2010.

[12] I. Anderson and H. Muller, "Context Awareness via GSM Signal Strength Fluctuation," in Pervasive 2006, Late Breaking Results, 2006.

[13] T. Sohn, A. Varshavsky, A. Lamarca, M. Y. Chen, T. Choudhury, I. Smith, S. Consolvo, J. Hightower, W. G. Griswold, and E. D. Lara, "Mobility Detection Using Everyday GSM Traces," in Ubicomp '06: Proceedings of the Eighth International Conference on Ubiquitous Computing, 2006, pp. 212-224.

[14] M. Youssef, M. Mah, and A. Agrawala, "Challenges: Device-free Passive Localization for Wireless Environments," in MobiCom '07: Proceedings of the 13th Annual ACM International Conference on Mobile Computing 
and Networking. ACM, 2007, pp. 222-229.

[15] M. Moussa and M. Youssef, "Smart Devices for Smart Environments: Device-free Passive Detection in Real Environments," in IEEE PerCom Workshops, 2009.

[16] A. E. Kosba, A. Abdelkader, and M. Youssef, "Analysis of a Device-free Passive Tracking System in Typical Wireless Environments ," in NTMS '09: Proceedings of the 3rd International Conference on New Technologies, Mobility and Security, 2009, pp. 291-295.

[17] M. Seifeldin and M. Youssef, "A Deterministic Large-scale Device-free Passive Localization System for Wireless Environments," in PETRA '10: Proceedings of the 3rd International Conference on Pervasive Technologies Related to Assistive Environments, 2010.

[18] M. A. Seifeldin, A. F. El-keyi, and M. A. Youssef, "Kalman filter-based tracking of a device-free passive entity in wireless environments," in WiNTECH'11: Proceedings of the 6th ACM International Workshop on Wireless Network Testbeds, Experimental Evaluation and Characterization. New York, NY, USA: ACM, 2011, pp. 43-50.

[19] J. Krumm, S. Harris, B. Meyers, B. L. Brumitt, M. Hale, and S. A. Shafer, "Multi-Camera Multi-Person Tracking for Easyliving," in Proceedings of the Third IEEE International Workshop on Visual Surveillance, 2000, pp. 3-10.

[20] J. Wilson and N. Patwari, "Radio Tomographic Imaging with Wireless Networks," IEEE Transactions on Mobile Computing, May 2010.

[21] R. J. Orr and G. D. Abowd, "The Smart Floor: A Mechanism for Natural User Identification and Tracking," in Proceedings of ACM CHI 2000 Conference on Human Factors in Computing Systems, vol. 2, 2000.

[22] S. S. Ram, Y. Li, A. Lin, and H. Ling, "Human Tracking Using Doppler Processing and Spatial Beamforming." IEEE Radar Conference, 2007.

[23] D. Zhang, J. Ma, Q. Chen, and L. M. Ni, "An RF-Based System for Tracking Transceiver-Free Objects," in PerCom '07: Proceedings of the Fifth IEEE International Conference on Pervasive Computing and Communications, 2007, pp. 135-144.

[24] R. S. Moore, R. Howard, P. Kuksa, and R. P. Martin, "A Geometric Approach to Device-Free Motion Localization Using Signal Strength," Rutgers University, Tech. Rep. DCS-TR-674, September 2010.

[25] A. L. AlHusseiny, M. Youssef, and M. ELTowiessy, "WCPS-OSL: A wireless cyber-physical system form object sensing and localization," in The Workshop on Collaborative, Autonomic, and Resilient Defenses for Cyber Physical Systems (CyPhyCARD'11), in conjunction with CollaborateCom 2011.

[26] N. Kassem, A. E. Kosba, and M. Youssef, "RF-based vehicle detection and speed estimation," in VTC'12-Spring: Proceedings of the 75th IEEE Vehicular Technology Conference, 2012.

[27] D.-Y. Yeung and C. Chow, "Parzen-Window Network Intrusion Detectors," in ICPR '02: Proceedings of the Sixteenth International Conference on Pattern Recognition, 2002, pp. 385-388.

[28] C. M. Bishop, "Novelty Detection and Neural Network Validation," Vision, Image and Signal Processing, IEE Proceedings-, vol. 141, no. 4, pp. 217-222, 1994.

[29] S. Subramaniam, T. Palpanas, D. Papadopoulos, V. Kalogeraki, and D. Gunopulos, "Online Outlier Detection in Sensor Data Using Non-parametric Models," in VLDB '06: Proceedings of the 32nd International Conference on Very Large Data Bases, 2006, pp. 187-198.

[30] L. Su, W. Han, S. Yang, P. Zou, and Y. Jia, "Continuous Adaptive Outlier Detection on Distributed Data Streams," in Proceedings of the Third International Conference on High Performance Computing and Communications, 2007, pp. 74-85.

[31] B. W. Silverman, Density Estimation for Statistics and Data Analysis. Chapman \& Hall/CRC, April 1986.

[32] D. W. Scott, Multivariate Density Estimation: Theory, Practice, and Visualization. Wiley, 1992.

[33] A. Rahim, S. Zeisberg, M. Fernandez, and A. Finger, "Impact of People Movement on Received Signal in Fixed Indoor Radio Communications," in Proceedings of the 17th IEEE International Symposium on Personal, Indoor and Mobile Radio Communications, 2006.

[34] C. J. V. Rijsbergen, Information Retrieval, 2nd ed. Newton, MA, USA: Butterworth-Heinemann, 1979.

[35] W. Cochran, "The Distribution of Quadratic Forms in a Normal System with Applications to the Analysis of Covariance," in Mathematical Proceedings of the Cambridge Philosophical Society, 1934.

[36] K. Kaemarungsi and P. Krishnamurthy, "Modeling of Indoor Positioning Systems based on Location Fingerprinting," in INFOCOM 2004. Twenty-third Annual Joint Conference of the IEEE Computer and Communications Societies, vol. 2, pp. 1012-1022. 
[37] M. Youssef and A. Agrawala, "Handling samples correlation in the Horus system," in INFOCOM'04: Twenty-third Annual Joint Conference of the IEEE Computer and Communications Societies. 